\title{
Weak Baire measurability of the balls in a Banach space
}

\author{
by \\ José RodRíguez (Valencia)
}

\begin{abstract}
Let $X$ be a Banach space. The property $(\star)$ "the unit ball of $X$ belongs to Baire $\left(X\right.$, weak)" holds whenever the unit ball of $X^{*}$ is weak ${ }^{*}$-separable; on the other hand, it is also known that the validity of $(\star)$ ensures that $X^{*}$ is weak ${ }^{*}$-separable. In this paper we use suitable renormings of $\ell^{\infty}(\mathbb{N})$ and the Johnson-Lindenstrauss spaces to show that $(\star)$ lies strictly between the weak ${ }^{*}$-separability of $X^{*}$ and that of its unit ball. As an application, we provide a negative answer to a question raised by K. Musiał.
\end{abstract}

1. Introduction. There are several $\sigma$-algebras on a Banach space $X$, like the Borel $\sigma$-algebras associated to the weak $(w)$ and norm topologies, as well as the Baire $\sigma$-algebra $\operatorname{Baire}(X, w)$ associated to $w$. G. A. Edgar [2] showed that $\operatorname{Baire}(X, w)$ is exactly the smallest $\sigma$-algebra on $X$ for which each element of $X^{*}$ (the topological dual of $X$ ) is measurable. In general, we have

$$
\operatorname{Baire}(X, w) \subset \operatorname{Borel}(X, w) \subset \operatorname{Borel}(X, \operatorname{norm}) .
$$

Although this chain collapses for separable $X$, some of these inclusions may be strict beyond the separable case (see [2], [3] and [15]).

Let $\|\cdot\|$ be an equivalent norm on $X$ and denote by $\|\cdot\|^{*}$ the corresponding equivalent norm on $X^{*}$. Clearly, the unit ball $B(X,\|\cdot\|)=\{x \in X:\|x\| \leq 1\}$ belongs to $\operatorname{Baire}(X, w)$ provided that $B\left(X^{*},\|\cdot\|^{*}\right)$ is separable for the weak* $\left(w^{*}\right)$ topology (equivalently, $(X,\|\cdot\|)$ is isometric to a subspace of $\ell^{\infty}(\mathbb{N})$ ), because in this case

$$
B(X,\|\cdot\|)=\bigcap_{x^{*} \in D}\left\{x \in X:\left|x^{*}(x)\right| \leq 1\right\}
$$

for any countable $w^{*}$-dense set $D \subset B\left(X^{*},\|\cdot\|^{*}\right)$. On the other hand, it is known (cf. [10, Theorem 1.5.3]) that the statement " $B(X,\|\cdot\|) \in$

2000 Mathematics Subject Classification: 28A05, 28B05, 46B20, 46G10.

Key words and phrases: Banach space, weak ${ }^{*}$-separability, Baire $\sigma$-algebra, scalar measurability, Pettis integral.

This research was partially supported by MEC (Spain) and FEDER, project MTM2005-08379, and Fundación Séneca (Spain), project 00690/PI/04. 
$\operatorname{Baire}(X, w)$ " (equivalent to saying that the mapping $\|\cdot\|: X \rightarrow \mathbb{R}$ is Baire $(X, w)$-measurable) implies that $X^{*}$ is $w^{*}$-separable. In general, the $w^{*}$-separability of $X^{*}$ is not sufficient to ensure the $w^{*}$-separability of $B\left(X^{*},\|\cdot\|^{*}\right)$ (see the next paragraph), so it is natural to ask whether the statement " $B(X,\|\cdot\|) \in \operatorname{Baire}(X, w)$ " is equivalent to the $w^{*}$-separability of $B\left(X^{*},\|\cdot\|^{*}\right)$ or that of $X^{*}$. We stress that the weak Baire measurability of the norm has important consequences in vector integration (see [6], [13] and [14]).

The aim of this paper is to discuss the question above. We use some ideas of G. A. Edgar (from [3] and [16]) to construct suitable equivalent norms on the following Banach spaces with $w^{*}$-separable dual: $\ell^{\infty}(\mathbb{N})$ and the JohnsonLindenstrauss spaces $J L_{0}$ and $J L_{2}$ [11] (see Section 2 for the definitions). In this way, for each of these spaces $X$ we find an equivalent norm $\|\cdot\|$ such that $B(X,\|\cdot\|)$ does not belong to Baire $(X, w)$ (Theorem 2.3). This improves the well known fact that each of these spaces admits an equivalent norm whose dual unit ball is not $w^{*}$-separable; see [5] (and [4, Theorem 12.58 and Exercise 12.40]) for $\ell^{\infty}(\mathbb{N})$ and $J L_{0}$, and [11] for $J L_{2}$ (in fact, no norm on this space has $w^{*}$-separable dual unit ball). Incidentally, the proof of Theorem 2.3 provides an example of an $X$-valued Pettis integrable function $f$ for which there is no scalarly equivalent $X$-valued function $g$ such that the mapping $\|g(\cdot)\|$ is measurable (Corollary 2.4). This answers negatively a question posed by K. Musiał [12, Problem 4]. Finally, the previous constructions also allow us to show that there is an equivalent norm $\|\cdot\|$ on $\ell^{\infty}(\mathbb{N})$ such that $B\left(\ell^{\infty}(\mathbb{N}),\|\cdot\|\right)$ belongs to Baire $\left(\ell^{\infty}(\mathbb{N}), w\right)$ but $B\left(\ell^{\infty}(\mathbb{N})^{*},\|\cdot\|^{*}\right)$ is not $w^{*}$-separable (Theorem 2.9).

For all unexplained terminology and notation we refer the reader to our standard references [4], [12] and [16]. The cardinality of the continuum is denoted by $\mathfrak{c}$ and the first uncountable ordinal by $\omega_{1}$. Let $X$ be a Banach space. Given $x^{*} \in X^{*}$ and $x \in X$, we write either $x^{*}(x)$ or $\left\langle x^{*}, x\right\rangle$ to denote the evaluation of $x^{*}$ at $x$. We say that a function $f: \Omega \rightarrow X$ defined on a complete probability space $(\Omega, \Sigma, \mu)$ is

(i) scalarly measurable if the composition $\left\langle x^{*}, f\right\rangle$ is $\Sigma$-measurable for every $x^{*} \in X^{*}$ (equivalently, $f$ is $\Sigma$-Baire $(X, w)$-measurable);

(ii) Pettis integrable if $\left\langle x^{*}, f\right\rangle$ is $\mu$-integrable for every $x^{*} \in X^{*}$ and for each $E \in \Sigma$ there is $x_{E} \in X$ such that $\int_{E}\left\langle x^{*}, f\right\rangle d \mu=\left\langle x^{*}, x_{E}\right\rangle$ for every $x^{*} \in X^{*}$.

Two functions $f, g: \Omega \rightarrow X$ are said to be scalarly equivalent if for each $x^{*} \in X^{*}$ we have $\left\langle x^{*}, f\right\rangle=\left\langle x^{*}, g\right\rangle \mu$-a.e. (the null set depends on $x^{*}$ ). Recall that $X$ has the property $(C)$ of Corson if every family of convex closed subsets of $X$ with empty intersection contains a countable subfamily with empty intersection. 
2. The results. In order to recall the definition of the Johnson-Lindenstrauss spaces [11] we need to introduce the following notation:

- $T_{n}:=\{0,1\}^{n}$ for every $n \in \mathbb{N}$;

- $T:=\bigcup_{n=1}^{\infty} T_{n}$;

- $K:=\{0,1\}^{\mathbb{N}}$;

- $u \mid n:=\left(u_{i}\right)_{i=1}^{n} \in T_{n}$ for every $u=\left(u_{i}\right)_{i=1}^{\infty} \in K$ and every $n \in \mathbb{N}$;

- $B_{u}:=\{u \mid n: n \in \mathbb{N}\} \subset T$ for every $u \in K$.

Note that $T$ is infinite countable and that (i) each $B_{u}$ is infinite; (ii) $B_{u} \cap B_{u^{\prime}}$ is finite whenever $u \neq u^{\prime}$; and (iii) the family $\left\{B_{u}: u \in K\right\}$ has cardinality $\mathfrak{c}$. The existence of a family of subsets of $\mathbb{N}$ satisfying (i)-(iii) was first proved by Sierpiński (cf. [4, Lemma 5.16]). We isolate this fact for future reference.

Lemma 2.1. Let $A$ be an infinite countable set. Then there is a family $\left\{A_{\gamma}: \gamma<\mathfrak{c}\right\}$ of infinite subsets of $A$ such that $A_{\gamma} \cap A_{\gamma^{\prime}}$ is finite whenever $\gamma \neq \gamma^{\prime}$.

As usual, for each $A \subset T$ we write $\chi_{A} \in \ell^{\infty}(T)$ to denote the characteristic function of $A$. Let $U_{0}$ be the linear span of $c_{0}(T) \cup\left\{\chi_{B_{u}}: u \in K\right\}$ in $\ell^{\infty}(T)$. Any $x \in U_{0}$ can be written in a unique way as $x=y+\sum_{i=1}^{p} a_{i} \chi_{B_{u_{i}}}$, where $y \in c_{0}(T),\left\{u_{1}, \ldots, u_{p}\right\} \subset K$ and $a_{i} \in \mathbb{R}$ for all $1 \leq i \leq p$. For such an $x$, set

$$
\|x\|_{J L_{2}}:=\max \left\{\|x\|_{\infty},\left(\sum_{i=1}^{p}\left|a_{i}\right|^{2}\right)^{1 / 2}\right\} .
$$

$\left(U_{0},\|\cdot\|_{J L_{2}}\right)$ is a normed space whose completion will be denoted by $\left(J L_{2},\|\cdot\|_{J L_{2}}\right)$; this is the Banach space $U$ studied in [11, Example 1]. On the other hand, the closure $J L_{0}$ of $U_{0}$ in $\ell^{\infty}(T)$ is a Banach space when equipped with $\|\cdot\|_{\infty}$; this space was discussed in [11, Example 2]. Our notation for the Johnson-Lindenstrauss spaces comes from [17], where the reader can find a lot of information on the role played by these spaces in Banach space theory.

In the proofs of Theorems 2.3 and 2.9 we will use the following norm introduced by Edgar in his example [16, Example 3-3-5] (cf. [12, Example 3.4]) of a scalarly bounded function which is not scalarly equivalent to a bounded function.

Definition 2.2. For each $u \in K$, consider the seminorm $\|\cdot\|_{u}$ on $\ell^{\infty}(T)$ given by

$$
\|x\|_{u}:=\limsup _{n \rightarrow \infty}\left|x_{u \mid n}\right|, \quad x \in \ell^{\infty}(T) .
$$

Let $a: K \rightarrow[1, \infty)$ be a bounded function. Define

$$
\|x\|_{a}:=\max \left\{\|x\|_{\infty}, \sup _{u \in K} a(u)\|x\|_{u}\right\}, \quad x \in \ell^{\infty}(T) .
$$

Clearly, $\|\cdot\|_{a}$ is an equivalent norm on $\ell^{\infty}(T)$. 
We write $\left(K, \Sigma_{K}, \mu_{K}\right)$ to denote the complete probability space obtained by completing the usual product probability measure on $\operatorname{Borel}(K)$. Recall that this measure space is isomorphic to $[0,1]$ equipped with the Lebesgue measure on the $\sigma$-algebra of all Lebesgue measurable sets (cf. [8, 254K]).

Theorem 2.3. Let $X$ be either $\ell^{\infty}(\mathbb{N}), J L_{0}$ or $J L_{2}$. Then $X^{*}$ is $w^{*}-$ separable and there is an equivalent norm $\|\cdot\|$ on $X$ such that $B(X,\|\cdot\|)$ does not belong to $\operatorname{Baire}(X, w)$.

Proof. The first assertion is obvious for $\ell^{\infty}(T)$ and its closed subspace $J L_{0}$. The $w^{*}$-separability of $J L_{2}^{*}$ was proved in [11, Example 1].

Now fix a bounded non- $\Sigma_{K}$-measurable function $a: K \rightarrow[1, \infty)$.

First case: $J L_{2}$. The identity mapping on $U_{0}$ can be extended to a continuous linear mapping $S: J L_{2} \rightarrow J L_{0}$. Note that the formula

$$
\|z\|:=\|z\|_{J L_{2}}+\|S(z)\|_{a}, \quad z \in J L_{2},
$$

defines an equivalent norm on $J L_{2}$. On the other hand, Edgar showed in the proof of $[3$, Proposition 5.12(c) $]$ that the function

$$
\phi: K \rightarrow J L_{2}, \quad \phi(u):=\chi_{B_{u}},
$$

is scalarly measurable, i.e. $\Sigma_{K}$-Baire $\left(J L_{2}, w\right)$-measurable. For each $u \in K$ we have $\left\|\chi_{B_{u}}\right\|_{a}=a(u)$, hence $\|\phi(u)\|=1+a(u)$. Since $a$ is not $\Sigma_{K}$-measurable, the mapping $\|\cdot\|: J L_{2} \rightarrow \mathbb{R}$ cannot be $\operatorname{Baire}\left(J L_{2}, w\right)$-measurable.

Second case: $J L_{0}$ and $\ell^{\infty}(T)$. Clearly, the composition $S \circ \phi: K \rightarrow J L_{0}$ is also scalarly measurable, i.e. $\Sigma_{K}$-Baire $\left(J L_{0}, w\right)$-measurable, and $\|(S \circ \phi)(u)\|_{a}$ $=a(u)$ for every $u \in K$. It follows that the restriction of $\|\cdot\|_{a}$ to $J L_{0}$ is not Baire $\left(J L_{0}, w\right)$-measurable. Finally, since

$$
\operatorname{Baire}\left(J L_{0}, w\right)=\left\{C \cap J L_{0}: C \in \operatorname{Baire}\left(\ell^{\infty}(T), w\right)\right\},
$$

we infer that $\|\cdot\|_{a}$ is not $\operatorname{Baire}\left(\ell^{\infty}(T), w\right)$-measurable. The proof is complete.

Let $(\Omega, \Sigma, \mu)$ be a complete probability space and $(X,\|\cdot\|)$ a Banach space. Musial posed in [12, Problem 4] the following question. Is it true that for each Pettis integrable function $f: \Omega \rightarrow X$ there is a function $g: \Omega \rightarrow X$ such that $f$ and $g$ are scalarly equivalent and the mapping $\|g(\cdot)\|$ is $\Sigma$-measurable? Naturally, the answer is affirmative if $(X, w)$ is measure compact (e.g. Lindelöf), since in this case every scalarly measurable $X$-valued function is scalarly equivalent to a strongly measurable one [2]. The following corollary provides a negative answer to Musiał's question even for spaces with property (C) (like $J L_{0}$ and $J L_{2}$, cf. [17, Section 2]). 
Corollary 2.4. Let $X$ be either $\ell^{\infty}(\mathbb{N}), J L_{0}$ or $J L_{2}$. Then there exist an equivalent norm $\|\cdot\|$ on $X$ and a Pettis integrable function $f: K \rightarrow X$ for which there is no function $g: K \rightarrow X$ such that $f$ and $g$ are scalarly equivalent and the mapping $\|g(\cdot)\|$ is $\Sigma_{K}$-measurable.

Proof. We first deal with $J L_{2}$. Let $f:=\phi$ be the function considered in the proof of Theorem 2.3. Since $J L_{2}$ has property (C) and $f$ is bounded and scalarly measurable, we can apply [16, Theorem 5-2-2] to conclude that $f$ is Pettis integrable. As shown in the proof of Theorem 2.3, there is an equivalent norm $\|\cdot\|$ on $J L_{2}$ such that the mapping $\|f(\cdot)\|$ is not $\Sigma_{K}$-measurable. Now, if a function $g: K \rightarrow J L_{2}$ is scalarly equivalent to $f$, the $w^{*}$-separability of $J L_{2}^{*}$ ensures that $f=g \mu_{K}$-a.e., and therefore $\|g(\cdot)\|$ is not $\Sigma_{K}$-measurable either.

The proof for $J L_{0}$ and $\ell^{\infty}(T)$ is similar, with the use of the Pettis integrable function $f:=S \circ \phi$.

Remark 2.5. A. S. Granero et al. [9] have shown that any Banach space $X$ without property $(\mathrm{C})$ admits an equivalent norm $\|\cdot\|$ such that $B\left(X^{*},\|\cdot\|^{*}\right)$ is not $w^{*}$-separable (see [1] for related results). In general, the failure of property $(\mathrm{C})$ does not ensure the existence of an equivalent norm $\|\cdot\|$ such that $B(X,\|\cdot\|) \notin \operatorname{Baire}(X, w)$. For instance, $\ell^{1}\left(\omega_{1}\right)$ fails to have property (C) [3] and

$$
\operatorname{Baire}\left(\ell^{1}\left(\omega_{1}\right), w\right)=\operatorname{Borel}\left(\ell^{1}\left(\omega_{1}\right), \operatorname{norm}\right),
$$

according to a theorem of D. H. Fremlin [7].

In Proposition 2.8 below we study the $w^{*}$-separability of $B\left(\ell^{\infty}(T)^{*},\|\cdot\|_{a}^{*}\right)$ in terms of $a$. To this end we need a couple of lemmas. The first one follows easily from the "lifting property" of $\ell^{1}(\mathbb{N})$ (cf. [4, Proposition 5.10]). As usual, we write

$$
c_{0}(T)^{\perp}:=\left\{x^{*} \in \ell^{\infty}(T)^{*}:\left\langle x^{*}, x\right\rangle=0 \text { for every } x \in c_{0}(T)\right\} .
$$

LEMMA 2.6. There is a decomposition $\ell^{\infty}(T)^{*}=Y \oplus c_{0}(T)^{\perp}$, where $Y$ is isomorphic to $\ell^{1}(T)$. The isomorphism $\Theta: \ell^{1}(T) \rightarrow Y$ is given by

$$
\langle\Theta(z), x\rangle=\sum_{u \mid n \in T} z_{u \mid n} x_{u \mid n}, \quad z \in \ell^{1}(T), x \in \ell^{\infty}(T),
$$

and the projection $P: \ell^{\infty}(T)^{*} \rightarrow Y$ is given by

$$
P\left(x^{*}\right)=\Theta\left(\left(x^{*}\left(\chi_{\{u \mid n\}}\right)\right)_{u \mid n \in T}\right), \quad x^{*} \in \ell^{\infty}(T)^{*} .
$$

Let $a: K \rightarrow[1, \infty)$ be a bounded function. If $\ell^{\infty}(T)^{*}$ is equipped with $\|\cdot\|_{a}^{*}$ and $\ell^{1}(T)$ is equipped with its canonical norm $\|\cdot\|_{\ell^{1}(T)}$, then $\Theta, \Theta^{-1}$ and $P$ each have norm 1. 
The second lemma isolates a property used by J. Hagler in his example (cf. [16, Example 3-2-4] or [12, Example 3.3]) of a scalarly measurable function which is not scalarly equivalent to a strongly measurable one. The original proof for the family $\left\{B_{u}: u \in K\right\}$ can be extended straightforwardly to this more general case.

Lemma 2.7. Let $\left\{C_{i}: i \in I\right\}$ be a family of subsets of $T$ such that $C_{i} \cap C_{i^{\prime}}$ is finite whenever $i \neq i^{\prime}$. Let $x^{*} \in c_{0}(T)^{\perp}$. Then the set $\left\{i \in I: x^{*}\left(\chi_{C_{i}}\right) \neq 0\right\}$ is countable.

Proposition 2.8. Let $a: K \rightarrow[1, \infty)$ be a bounded function. The following statements are equivalent:

(i) $B\left(\ell^{\infty}(T)^{*},\|\cdot\|_{a}^{*}\right)$ is $w^{*}$-separable.

(ii) $a(u)=1$ for every $u \in K$.

Proof. Clearly, (ii) implies that $\|\cdot\|_{a}=\|\cdot\|_{\infty}$, so it only remains to prove (i) $\Rightarrow$ (ii). Suppose that (ii) fails, that is, there is $u \in K$ such that $a(u)>1$. Take any countable set $D \subset B\left(\ell^{\infty}(T)^{*},\|\cdot\|_{a}^{*}\right)$. Since $B_{u}$ is infinite countable, we can find a family $\left\{A_{\gamma}: \gamma<\mathfrak{c}\right\}$ of infinite subsets of $B_{u}$ such that $A_{\gamma} \cap A_{\gamma^{\prime}}$ is finite whenever $\gamma \neq \gamma^{\prime}$ (Lemma 2.1). With the notations of Lemma 2.6, for each $x^{*} \in D$ we can write $x^{*}=P\left(x^{*}\right)+\left(x^{*}-P\left(x^{*}\right)\right)$, where $x^{*}-P\left(x^{*}\right) \in c_{0}(T)^{\perp}, P\left(x^{*}\right) \in Y$ and $\left\|P\left(x^{*}\right)\right\|_{a}^{*} \leq 1$. Since $D$ is countable, we can apply Lemma 2.7 to find $\gamma<\mathfrak{c}$ such that $x^{*}\left(\chi_{A_{\gamma}}\right)=P\left(x^{*}\right)\left(\chi_{A_{\gamma}}\right)$ for every $x^{*} \in D$. By the definition of $P$, we have

$$
P\left(x^{*}\right)\left(\chi_{A_{\gamma}}\right)=\sum_{u \mid n \in A_{\gamma}} x^{*}\left(\chi_{\{u \mid n\}}\right) .
$$

On the other hand, since $\Theta^{-1}$ has norm 1 , we get

$$
\sum_{v \mid n \in T}\left|x^{*}\left(\chi_{\{v \mid n\}}\right)\right|=\left\|\Theta^{-1}\left(P\left(x^{*}\right)\right)\right\|_{\ell^{1}(T)} \leq 1 .
$$

It follows that $\left|x^{*}\left(\chi_{A_{\gamma}}\right)\right| \leq 1$ for every $x^{*} \in D$. Since $A_{\gamma} \subset B_{u}$ is infinite, we conclude that

$$
\left\|\chi_{A_{\gamma}}\right\|_{a}=a(u)>1 \geq \sup _{x^{*} \in D}\left|x^{*}\left(\chi_{A_{\gamma}}\right)\right| .
$$

Therefore, $D$ is not $w^{*}$-dense in $B\left(\ell^{\infty}(T)^{*},\|\cdot\|_{a}^{*}\right)$. This shows that the ball $B\left(\ell^{\infty}(T)^{*},\|\cdot\|_{a}^{*}\right)$ is not $w^{*}$-separable.

TheOREM 2.9. There is an equivalent norm $\|\cdot\|$ on $\ell^{\infty}(\mathbb{N})$ such that:

(i) $B\left(\ell^{\infty}(\mathbb{N}),\|\cdot\|\right)$ belongs to $\operatorname{Baire}\left(\ell^{\infty}(\mathbb{N}), w\right)$.

(ii) $B\left(\ell^{\infty}(\mathbb{N})^{*},\|\cdot\|^{*}\right)$ is not $w^{*}$-separable. 
Proof. Fix a bounded function $a: K \rightarrow[1, \infty)$ such that $a^{-1}((1, \infty))$ is countable and non-empty. Then $B\left(\ell^{\infty}(T)^{*},\|\cdot\|_{a}^{*}\right)$ is not $w^{*}$-separable, by Proposition 2.8. On the other hand, note that for each $u \in K$ the mapping

$$
\|\cdot\|_{u}: \ell^{\infty}(T) \rightarrow \mathbb{R}, \quad\|x\|_{u}=\limsup _{n \rightarrow \infty}\left|x_{u \mid n}\right|=\inf _{k \in \mathbb{N}} \sup _{n \geq k}\left|x_{u \mid n}\right|,
$$

is $\operatorname{Baire}\left(\ell^{\infty}(T), w\right)$-measurable. Since

$$
\|x\|_{a}=\max \left\{\|x\|_{\infty}, \sup _{u \in a^{-1}((1, \infty))} a(u)\|x\|_{u}\right\}, \quad x \in \ell^{\infty}(T),
$$

it follows that $\|\cdot\|_{a}$ is $\operatorname{Baire}\left(\ell^{\infty}(T), w\right)$-measurable, as required.

Acknowledgements. The author wishes to express his gratitude to Susumu Okada for suggesting the problem considered in this paper.

\section{References}

[1] J. M. Borwein and J. D. Vanderwerff, Constructible convex sets, Set-Valued Anal. 12 (2004), 61-77.

[2] G. A. Edgar, Measurability in a Banach space, Indiana Univ. Math. J. 26 (1977), 663-677.

[3] -, Measurability in a Banach space. II, ibid. 28 (1979), 559-579.

[4] M. Fabian, P. Habala, P. Hájek, V. Montesinos Santalucía, J. Pelant, and V. Zizler, Functional Analysis and Infinite-Dimensional Geometry, CMS Books in Math. 8, Springer, New York, 2001.

[5] C. Finet and G. Godefroy, Biorthogonal systems and big quotient spaces, in: Banach Space Theory (Iowa City, IA, 1987), Contemp. Math. 85, Amer. Math. Soc., Providence, RI, 1989, 87-110.

[6] D. H. Fremlin, The McShane and Birkhoff integrals of vector-valued functions, Univ. Essex Mathematics Department Research Report 92-10, version of 18.5.07 available at http://www.essex.ac.uk/maths/staff/fremlin/preprints.htm.

[7] —, Borel sets in nonseparable Banach spaces, Hokkaido Math. J. 9 (1980), 179-183.

[8] -, Measure Theory. Volume 2: Broad Foundations, Torres Fremlin, Colchester, 2001.

[9] A. S. Granero, M. Jiménez, A. Montesinos, J. P. Moreno, and A. Plichko, On the Kunen-Shelah properties in Banach spaces, Studia Math. 157 (2003), 97-120.

[10] M. Heiliö, Weakly summable measures in Banach spaces, Ann. Acad. Sci. Fenn. Ser. A I Math. Dissertationes 66 (1988).

[11] W. B. Johnson and J. Lindenstrauss, Some remarks on weakly compactly generated Banach spaces, Israel J. Math. 17 (1974), 219-230.

[12] K. Musiał, Topics in the theory of Pettis integration, Rend. Istit. Mat. Univ. Trieste 23 (1991), 177-262.

[13] J. Rodríguez, The Bourgain property and convex hulls, Math. Nachr. 280 (2007), 1302-1309.

[14] —, Convergence theorems for the Birkhoff integral, Houston J. Math., to appear.

[15] M. Talagrand, Comparaison des Boreliens d'un espace de Banach pour les topologies fortes et faibles, Indiana Univ. Math. J. 27 (1978), 1001-1004.

[16] —, Pettis integral and measure theory, Mem. Amer. Math. Soc. 51 (1984), no. 307. 
[17] V. Zizler, Nonseparable Banach spaces, in: Handbook of the Geometry of Banach Spaces, Vol. 2, North-Holland, Amsterdam, 2003, 1743-1816.

Departamento de Análisis Matemático Universidad de Valencia

Avda. Doctor Moliner 50

46100 Burjassot, Valencia, Spain
Current address: Instituto Universitario de Matemática Pura y Aplicada Universidad Politécnica de Valencia Camino de Vera $\mathrm{s} / \mathrm{n}$ 46022 Valencia, Spain

E-mail: jorodrui@mat.upv.es

Received January 29, 2007

Revised version December 21, 2007 